\title{
Higher-order statistics for stochastic electromagnetic interactions: applications to a thin-wire frame
}

\section{Citation for published version (APA):}

Sy, O. O., Beurden, van, M. C., Michielsen, B. L., Vaessen, J. A. H. M., \& Tijhuis, A. G. (2012). Higher-order statistics for stochastic electromagnetic interactions: applications to a thin-wire frame. Progress In Electromagnetics Research B, 41, 307-332. https://doi.org/10.2528/PIERB12042104

DOI:

10.2528/PIERB12042104

Document status and date:

Published: 01/01/2012

\section{Document Version:}

Publisher's PDF, also known as Version of Record (includes final page, issue and volume numbers)

\section{Please check the document version of this publication:}

- A submitted manuscript is the version of the article upon submission and before peer-review. There can be important differences between the submitted version and the official published version of record. People interested in the research are advised to contact the author for the final version of the publication, or visit the $\mathrm{DOI}$ to the publisher's website.

- The final author version and the galley proof are versions of the publication after peer review.

- The final published version features the final layout of the paper including the volume, issue and page numbers.

Link to publication

\section{General rights}

Copyright and moral rights for the publications made accessible in the public portal are retained by the authors and/or other copyright owners and it is a condition of accessing publications that users recognise and abide by the legal requirements associated with these rights.

- Users may download and print one copy of any publication from the public portal for the purpose of private study or research.

- You may not further distribute the material or use it for any profit-making activity or commercial gain

- You may freely distribute the URL identifying the publication in the public portal.

If the publication is distributed under the terms of Article 25fa of the Dutch Copyright Act, indicated by the "Taverne" license above, please follow below link for the End User Agreement:

www.tue.nl/taverne

Take down policy

If you believe that this document breaches copyright please contact us at:

openaccess@tue.nl

providing details and we will investigate your claim. 


\title{
HIGHER-ORDER STATISTICS FOR STOCHASTIC ELECTROMAGNETIC INTERACTIONS: APPLICATIONS TO A THIN-WIRE FRAME
}

\author{
O. O. $\mathrm{Sy}^{1, *}$, M. C. Van Beurden ${ }^{1}$, B. L. Michielsen ${ }^{2}$, \\ J. A. H. M. Vaessen ${ }^{1}$, and A. G. Tijhuis ${ }^{1}{ }^{\dagger}$
}

${ }^{1}$ Department of Electrical Engineering, Eindhoven University of Technology, Den Dolech 2, 5600 MB, Eindhoven, The Netherlands

${ }^{2}$ ONERA-DEMR, BP 74025, 2, av. Edouard Belin, 31055 Toulouse Cedex 4, France

\begin{abstract}
Uncertainties in an electromagnetic observable, that arise from uncertainties in geometric and electromagnetic parameters of an interaction configuration, are here characterized by combining computable higher-order moments of the observable with higher-order Chebychev inequalities. This allows for the estimation of the range of the observable by rigorous confidence intervals. The estimated range is then combined with the maximum-entropy principle to arrive at an efficient and reliable estimation of the probability density function of the observable. The procedure is demonstrated for the case of the induced voltage of a thin-wire frame that has a random geometry, is connected to a random load, and is illuminated by a random incident field.
\end{abstract}

\section{INTRODUCTION}

Various numerical methods have been developed to efficiently model interactions between electromagnetic waves and material systems. The method of moments (MoM), transmission-line methods or finite-difference schemes are just a few examples of such numerical methods $[1,2]$. These methods can all be regarded as deterministic "black boxes", which given the geometrical and physical properties of a material system, and the characteristics of the ambient electromagnetic

\footnotetext{
Received 21 April 2012, Accepted 8 June 2012, Scheduled 13 June 2012

* Corresponding author: Ousmane Oumar Sy (ousmane.o.sy@jpl.nasa.gov).

$\dagger$ O. O. Sy is now at Jet Propulsion Laboratory, California Institute of Technology, Pasadena, California.
} 
field, compute observables ranging from network parameters to antenna and scattering parameters.

However, the study of real-life interactions often requires the consideration of multiple configurations. Further, in many cases, the actual configuration is not entirely known due to a lack of information or due to measurement errors. This variability is particularly significant when dealing with highly sensitive models that depend on multiple input variables: a slight modification of the configuration may produce large variations of the observable, as is the case with resonant structures.

A brute-force deterministic approach consisting in the systematic execution of the model for every possible configuration is generally ruled out by the cost implied by even a single execution of the deterministic model and by the possibly large number of configurations to analyze. These limitations motivate the use of a stochastic approach, in which the configuration is assumed to vary randomly according to a given probability distribution known a priori. As a result, the observable becomes random and follows a probability distribution that is yet unknown. Probability theory then provides a wide selection of tools to characterize the distribution of the observable either partially via statistical moments, or completely through its probability density function (pdf) or its cumulative distribution function (cdf).

The range of applications of stochastic uncertainty-quantification methods is broad and spans from random media propagation [3], to bio-electromagnetics [4], antenna design [5] and electromagnetic compatibility (EMC) [6]. In the latter domain, statistical methods are commonly used to describe the field and power distributions in mode-stirred reverberation chambers $[7,8]$. Thin-wire setups, which are ubiquitous as interconnections in modern-day electronics, are also more and more prone to being analyzed stochastically $[9,10]$. We have successfully applied this modus operandi to thin-wire problems by evaluating the first four statistical moments of the observable with the aid of quadrature algorithms. The thus obtained statistics could be post-processed using Chebychev's inequality to obtain general bounds of the distribution of the observable via its second-order moments [11] or to quantify the likelihood of observing extreme samples using the fourth-order moment, or kurtosis [12].

Although the second-order bounds hold for any random observable with finite second-order moments, these bounds are in essence very loose. Moreover, the use of the kurtosis as an indication of the presence of outliers was performed by comparing the value of the kurtosis to the reference value of 3, which corresponds to a Gaussian distribution [13, p. 148]. This analysis did not quantify the probability 
of observing such outliers, nor did it indicate the distance from the mean at which to expect these outliers. In this article, we will address these shortcomings by resorting to higher-order moments and to higher-order Chebychev inequalities. In addition to refining the bounds on the probability distribution of the observable, this approach allows for the estimation of the range of the observable by accurately computed confidence intervals.

While higher-order moments reveal finer features about the randomness of the observable, the ultimate goal is to determine the distribution of the observable. To this end, the Maximum-Entropy (MaxEnt) principle is particularly useful as it uses a sequence of statistical moments to approximate the pdf of the observable [14]. However, to be applicable the MaxEnt principle requires the knowledge of the range of the observable, which is not granted when dealing with deterministic "black-box" models like the ones considered in this paper. We will overcome this issue by using the estimates of the observable's range derived from the higher-order Chebychev inequalities. The resulting MaxEnt pdf will thus provide the highest level of information regarding the randomness of the observable. To the best of the authors' knowledge, this is the first stochastic approach which uses Chebychev's higher-order inequalities to approximate the range of the observable and then use this range estimate in a MaxEnt post-processing.

The outline of this article is as follows. Subsection 2.1 describes the deterministic model used to represent the voltage induced at the port of a thin-wire setup by an incident electromagnetic field. A stochastic formalism is then adopted in Subsection 2.2 to account for uncertainties in the interaction configuration and in the induced voltage, which is the observable. As a result, statistical moments of the observable can be computed via quadrature rules as explained in Subsection 2.3. With these statistical moments at hand, higherorder Chebychev inequalities are employed in Section 3 to bound the probability distribution and the range of the observable. Next, in Section 4, the use of the estimates of the range of the observable in a maximum-entropy approach is described. The stochastic rationale is then illustrated in Section 5 through the study of the voltage induced by a random plane wave at the port of a thin-wire system that has a randomly varying geometry and is connected to a random resistance. Finally, conclusions are drawn in Section 6. 


\section{STOCHASTIC MODEL}

\subsection{Deterministic Description of the Setup}

The coupling between a thin-wire setup and an incident electromagnetic field is a classical test case in EMC [15]. This problem is relevant for the analysis of the electromagnetic interference signals induced in cables that connect devices such as control computers and actuators, e.g., in buildings, in automobiles or in aircrafts [16]. In many cases of practical interest, these interconnecting cables are hidden from view being either buried or embedded inside walls of buildings or in the fuselage of an aircraft. Further, even if a very precise cable routing CAD tool is used during the design, the degree of imprecision of operators installing the cables will lead to a priori installation uncertainties. Hence, in all these cases the exact geometrical layout of the wire is generally not known in detail, which is a problem in a posteriori modeling.

A generic model of electromagnetic coupling in free space between a thin-wire system and a time-harmonic incident electromagnetic field is sketched in Fig. 1. The configuration involves a thin wire $\Omega_{\alpha}$ located above an infinite perfectly electrically conducting (PEC) ground plane. The wire has a circular cross section with a diameter of $1 \mathrm{~mm}$ and, over a distance of $1 \mathrm{~m}$, a variable shape described by its Cartesian coordinates $\left[x_{\alpha}(y), y, z_{\alpha}(y)\right]$, for any $y \in\left[y_{m} ; y_{M}\right]$. The vector $\alpha$, which describes the geometry of $\Omega_{\alpha}$, belongs to the known domain $\mathcal{A} \subset \mathbb{R}^{L}$. This device possesses two port regions that have negligible dimensions compared to the wavelength. One of the port regions is connected to a resistance denoted $R \in \mathcal{R} \subset \mathbb{R}_{+}$, while the other port is in an opencircuit state. The parameters of the incident electric field $\mathbf{E}_{\beta}^{i}$, such as its amplitude, its polarization $\psi$, or its direction of propagation $(\theta, \phi)$ in a spherical coordinate system, are defined through the vector $\beta$, which belongs to the known domain $\mathcal{B} \subset \mathbb{R}^{M}$.

The electromagnetic coupling is monitored via the observable $\gamma=\left|V_{e}\right|$, which corresponds to the magnitude of the voltage induced at the open-circuit port of the wire. In a shielding context, $V_{e}$ represents an equivalent Thévenin voltage and indicates the susceptibility of the setup to fields induced at its port by external sources [17]. For any deterministic configuration $(\boldsymbol{\alpha}, \mathbf{R}, \boldsymbol{\beta})$, the observable is defined as

$$
\gamma(\boldsymbol{\alpha}, \mathbf{R}, \boldsymbol{\beta})=\left|V_{e}(\boldsymbol{\alpha}, \mathbf{R}, \boldsymbol{\beta})\right|=\frac{1}{I_{0}}\left|\int_{\mathbf{r} \in \Omega_{\boldsymbol{\alpha}}} \mathbf{J}_{\boldsymbol{\alpha}, R}(\mathbf{r}) \cdot \mathbf{E}_{\beta}^{i}(\mathbf{r}) d l(\mathbf{r})\right|,
$$

where the current distribution $\mathbf{J}_{\boldsymbol{\alpha}, R}$ is induced on $\Omega_{\boldsymbol{\alpha}}$ in the absence of $\mathbf{E}_{\boldsymbol{\beta}}^{i}$, when a current source $I_{0}$ is impressed at the open-circuit port of 


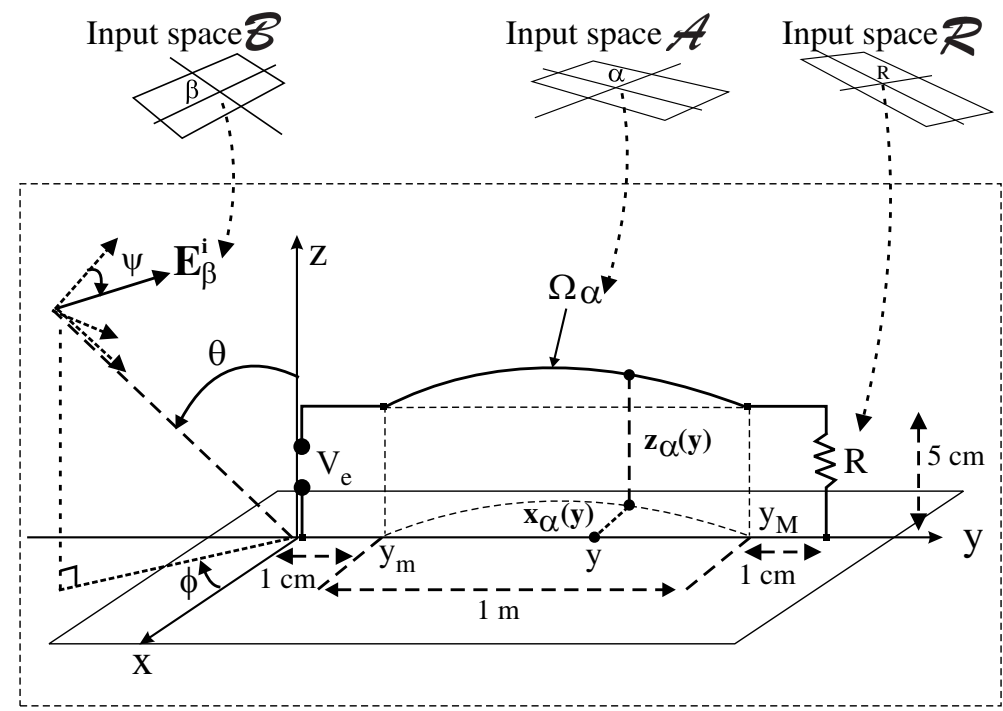

Interaction configuration

Figure 1. Interaction configuration: Thin wire $\Omega_{\boldsymbol{\alpha}}$ parameterized by the vector $\boldsymbol{\alpha} \in \mathcal{A}$, resistance $R \in \mathcal{R}$ and incident field $\mathbf{E}_{\boldsymbol{\beta}}^{i}$ parameterized by the vector $\boldsymbol{\beta}=\left(\theta, \phi, \psi,\left|\mathbf{E}_{\boldsymbol{\beta}}^{i}\right|\right) \in \mathcal{B}$.

the wire (left in Fig. 1) [18]. The line current $\mathbf{J}_{\boldsymbol{\alpha}, R}$ could be computed using transmission-line (TL) theory [19]. However, special care would be required to adapt the TL equations to the non-straight geometry of the wire $\Omega_{\boldsymbol{\alpha}}$, and to properly account for the radiation effects that occur along the line and in the load $R$ [20,21]. Instead, a full-wave model is used to obtain accurate results. A Pocklington electricfield integral equation (EFIE) associated with the transmitting state is solved by the method of moments to obtain $\mathbf{J}_{\boldsymbol{\alpha}, R}[22,23]$. This implies the computation of a full impedance matrix and the solution of the associated algebraic problem.

The definition of $V_{e}$ highlights the nonlinear dependence of the observable on the parameters of the configuration, since $\gamma(\boldsymbol{\alpha}, \mathbf{R}, \boldsymbol{\beta})$ depends on $\boldsymbol{\alpha}$ through $\mathbf{J}_{\boldsymbol{\alpha}, R}$ and the support $\Omega_{\boldsymbol{\alpha}}$ of the integral, on the resistance $R$ through the $\mathbf{J}_{\boldsymbol{\alpha}, R}$, while the effect of $\boldsymbol{\beta}$ appears through $\mathbf{E}_{\boldsymbol{\beta}}^{i}$. Further, for any given configuration $(\boldsymbol{\alpha}, \mathbf{R}, \boldsymbol{\beta})$, the evaluation of the associated response $\gamma(\boldsymbol{\alpha}, \mathbf{R}, \boldsymbol{\beta})$ involves a numerical cost, which stems from the establishment and the solution of the EFIE. 


\subsection{Uncertainties and Randomization}

The deterministic model described by Eq. (1) permits the computation of the observable for any given configuration $(\boldsymbol{\alpha}, \mathbf{R}, \boldsymbol{\beta})$. However, in the presence of uncertainties, the actual value of $(\boldsymbol{\alpha}, \mathbf{R}, \boldsymbol{\beta})$ is undetermined in $\mathcal{A} \times \mathcal{R} \times \mathcal{B}$.

The geometrical uncertainty of the wire translates into a variation of the total length of the thin wire. This model is mainly relevant for problems where the actual shape and length of the wire are not entirely known. The indetermination of the wire length has a major influence on the frequencies at which resonances occur [11]. In addition, the load $R$ influences strongly the presence of resonance as well as the value of the observable at those frequencies: while an adapted line will exhibit little or no resonance, a mismatched impedance will produce resonances with large quality factors, particularly if the line is terminated by an open- or short-circuit [21]. The varying polarization of $\mathbf{E}_{\beta}^{i}$ will also impact the amplitude of the observable around resonance frequencies [21]. Hence, the model used here is an example that shows many aspects of geometrical and field uncertainties at the same time. In this way, one captures both uncertain resonance frequencies, different exposures of the wires to the field and different load conditions.

By applying a stochastic rationale, the indetermination of $(\boldsymbol{\alpha}, \mathbf{R}, \boldsymbol{\beta})$ in $\mathcal{A} \times \mathcal{R} \times \mathcal{B}$ is assumed to be random. First, all the uncertain input parameters, i.e., the uncertain components of $(\boldsymbol{\alpha}, \mathbf{R}, \boldsymbol{\beta})$, are gathered in the vector $\mathbf{u}$, which belongs to the set $\mathcal{U} \subset \mathcal{A} \times \mathcal{R} \times \mathcal{B}$. For instance, if only the components $\alpha_{1}$ and $\beta_{1}$ are random, then the vector $\mathbf{u}=\left(\alpha_{1}, \beta_{1}\right)$ of random inputs will belong to the domain $\mathcal{A}_{1} \times \mathcal{B}_{1}$, which is a sub-domain of $\mathbb{R}^{2}$. The vector $\mathbf{u}$ is assumed to be randomly distributed in $\mathcal{U}$ according to a known probability distribution $P_{\mathcal{U}}$ that is chosen a priori. The choice of $P_{\mathcal{U}}$ is governed by the information available on the variations of $\mathbf{u}$ in $\mathcal{U}$.

Due to the randomness of $\mathbf{u}$, the observable $\gamma$, which is a function of $\mathbf{u}$, becomes random. To mark this induced randomness, the observable is written as $\gamma(\mathbf{u})$. Unlike $\mathbf{u}$, which is completely characterized by $P_{\mathcal{U}}$, the probability distribution $P_{\gamma}$ of $\gamma$ has yet to be determined. However, since the definition of $\gamma$ involves a numerical model, expressing $P_{\gamma}$ as a function of $P_{\mathcal{U}}$ is a non-trivial mathematical task $[24$, p. 36]. Hence, rather than aiming for a complete description of the randomness of $\gamma$ via $P_{\gamma}$, we focus on the statistical moments of $\gamma$, which are computable and still provide valuable information about the distribution of $\gamma$. 


\subsection{Computation of Statistical Moments}

The statistical moments of $\gamma$ are obtained by considering a measurable real-valued function $h$ defined on $\mathbb{R}$. The expectation $\mathbb{E}[h(\gamma)]$ of the random variable $h(\gamma)$ then reads [13, p. 209]

$$
\mathbb{E}[h(\gamma)]=\int_{\mathcal{U}} h\left[\gamma\left(\mathbf{u}^{\prime}\right)\right] f_{\mathcal{U}}\left(\mathbf{u}^{\prime}\right) d \mathbf{u}^{\prime},
$$

where $f_{\mathcal{U}}$ is the pdf of $\mathbf{u}$. The right-hand side of Eq. (2) can be determined numerically, since it consists of an integral over a computable integrand $h\left[\gamma\left(\mathbf{u}^{\prime}\right)\right] f_{\mathcal{U}}\left(\mathbf{u}^{\prime}\right)$ and a known domain of integration $\mathcal{U}$. With the aid of a quadrature rule $Q_{N}$, this integral is approximated as

$$
\mathbb{E}[h(\gamma)] \approx Q_{N}[h(\gamma)]=\sum_{n=1}^{N} h\left[\gamma\left(\mathbf{u}_{n}\right)\right] f_{\mathcal{U}}\left(\mathbf{u}_{n}\right) w_{n},
$$

where $N$ is the complexity of the quadrature rule. The abscissae $\mathbf{u}_{n} \in \mathcal{U}$ and the positive weights $w_{n}>0$, for $n=1, \ldots, N$, are defined by the type of quadrature algorithm employed [25, p. 114]. With stable quadrature rules, increasing $N$ improves the accuracy of the approximation in Eq. (3). However, $N$ also represents the number of evaluations of the deterministic model, which, as stated above, bears a certain cost. To limit the increase of $N$, nested quadrature rules are employed that allow for reusing samples of $\gamma$ to refine the mesh of the quadrature rule. Further, when multiple integrals need to be computed simultaneously, the same quadrature rule is employed. Moreover, the quadrature rule is chosen to efficiently handle integrals over the multidimensional domain $\mathcal{U}$, as is the case with a sparse-grid [26], a MonteCarlo [25, p. 205] or a space-filling-curve rule [11]. The convergence of the quadrature rule is monitored through the relative modification of $Q_{N}[h(\gamma)]$ as the number of samples $N$ is increased.

Following these guidelines, the first $M$ statistical moments of $\gamma$ are computed by quadrature using Eq. (3) and then gathered in the set

$$
\mathcal{M}(M)=\left\{\mu_{m}=\mathbb{E}\left[\gamma^{m}\right], m=0, \ldots, M\right\} .
$$

\section{HIGHER-ORDER CHEBYCHEV CONFIDENCE INTERVALS}

The knowledge of the range $\mathcal{G}$ of $\gamma$ is essential to determine the dynamic range of the observable in response to the uncertainties that affect the input variables $(\boldsymbol{\alpha}, \mathbf{R}, \boldsymbol{\beta})$. However, with an observable that is a 
nonlinear and non-monotonic function of the input variables $(\boldsymbol{\alpha}, \mathbf{R}, \boldsymbol{\beta})$, $\mathcal{G}$ can only be obtained by evaluating the deterministic model over the entire ensemble of possible configurations, viz. $\mathcal{G}=\gamma(\mathcal{A} \times \mathcal{R} \times \mathcal{B})$. The computational effort required by such an operation is often prohibitive for a numerical model. Instead, the statistical moments at hand, viz. $\mathcal{M}(M)$, can be employed together with the theory of probability to infer confidence domains, i.e., intervals in which the likelihood of observing the observable is quantified.

The confidence domains are chosen as intervals centered about the mean $\mu_{1}$ as follows $G(r)=\left[\mu_{1}-r \sigma ; \mu_{1}+r \sigma\right]$, where $\sigma=\sqrt{\mu_{2}-\mu_{1}^{2}}$ is the standard deviation and $r>0$. The confidence level $P_{\text {conf }}(r)$ and the exceedance probability $P_{\text {exc }}(r)$ associated with $G(r)$ are defined as

$$
\begin{aligned}
P_{\text {conf }}(r) & =P_{\gamma}[\gamma \in G(r)]=F_{\gamma}\left(\mu_{1}+r \sigma\right)-F_{\gamma}\left(\mu_{1}-r \sigma\right), \\
P_{\text {exc }}(r) & =P_{\gamma}\left(\left|\gamma-\mu_{1}\right|>r \sigma\right)=1-P_{\text {conf }}(r),
\end{aligned}
$$

where $F_{\gamma}$ is the cdf of $\gamma$. Exceedance probabilities are particularly valuable for risk and safety analyses. In an EMC context, $P_{\text {exc }}(r)$ indicates the likelihood of observing values of $\gamma=\left|V_{e}\right|$ more than $r \sigma$ away from the mean $\mu_{1}$, and therefore the intensity of the interferences induced at the port of the wire by the external incident field.

Without knowing $F_{\gamma}$, neither $P_{\text {conf }}(r)$ nor $P_{\text {exc }}(r)$ can be evaluated. The Gaussian estimate $P_{\text {Gauss }}(r)$ of $P_{\text {exc }}(r)$ can be obtained from Eq. (6) by replacing $F_{\gamma}$ by a Gaussian cdf $F_{\text {Gauss, }}$, with

$$
F_{\text {Gauss }}(r)=\frac{1}{2}\left[1+\operatorname{erf}\left(\frac{r-\mu_{1}}{\sqrt{2} \sigma}\right)\right], \quad \text { for any } r \in \mathbb{R} .
$$

However, without additional information, $P_{\gamma}$ cannot be assumed to be Gaussian, particularly when dealing with models that are not such that the Central-Limit Theorem applies [13, p. 278].

As an alternative, Chebychev's second-order inequality can be used to bound $P_{\text {exc }}(r)$ as it states that, for any $r>0,[13$, p. 151]

$$
P_{\text {exc }}(r)=P_{\gamma}\left(\left|\gamma-\mu_{1}\right|>r \sigma\right) \leq \frac{1}{r^{2}} .
$$

In other words, the interval $G(r)$ should contain at least $100 \times\left(1-1 / r^{2}\right) \%$ of the samples of $\gamma$. This type of asymptotic bound is very useful as it holds for any random variable with finite second-order moments. The inequality (8) is also rather conservative as it states, e.g., that $G(3)$ will contain at least $88.8 \%$ of the values of $\gamma$, whereas for Gaussian random variables $G(3)$ is known to contain more than $99.7 \%$ of the samples.

Chebychev's inequality can be refined to incorporate higherordered moments as follows

$$
P_{\text {exc }}(r) \leq \frac{\kappa_{2 n}}{r^{2 n}}=b_{2 n}(r)
$$


for any $n \in \mathbb{N} \backslash\{0\}$ and $r>0$ [13, p. 152]. The normalized moments $\kappa_{2 n}$ can be obtained from the moments $\mu_{n}$ about the origin via the correspondence

$$
\kappa_{2 n}=\mathbb{E}\left[\left(\frac{\gamma-\mu_{1}}{\sigma}\right)^{2 n}\right]=\frac{1}{\sigma^{2 n}} \sum_{\ell=0}^{2 n}\left(\begin{array}{c}
2 n \\
\ell
\end{array}\right)(-1)^{2 n-\ell} \mu_{\ell} \mu_{1}^{2 n-\ell} \geq 0 .
$$

These normalized moments are dimensionless since they correspond to the standardized variable $\widehat{\gamma}=\left(\gamma-\mu_{1}\right) / \sigma$. Chebychev's inequality (9) holds also for oddly ordered moments $\kappa_{2 n+1}=\mathbb{E}\left[\left|\gamma-\mu_{1}\right|^{2 n+1}\right] / \sigma^{2 n+1}$. However, due to the presence of the absolute value in their definition, the oddly ordered normalized moments can no longer be expressed in terms of the moments $\mu_{n}$ about the origin.

The right-hand side $b_{2 n}(r)$ of the inequality (9) is meaningful only once $b_{2 n}(r) \leq 1$, i.e., when $r \geq r_{\min }(2 n)=\kappa_{2 n}^{1 / 2 n}$. These minimum values of $r$ are ordered according to Lyapunov's inequality [13, p. 152], i.e.,

$$
\kappa_{2 n}^{1 / 2 n} \geq \kappa_{2(n-1)}^{1 / 2(n-1)} \geq \ldots \geq \kappa_{2}^{1 / 2}=1 .
$$

Hence, the higher the order of the Chebychev bound $b_{2 n}$, the larger the minimum length of the confidence interval $G\left[r_{\min }(2 n)\right]$. At the same time, the inequality (9) shows that as the length $r$ of $G(r)$ increases, $b_{2 n}(r)$ exhibits a much faster decay rate than $b_{2}(r)$, thereby leading to tighter bounds for $P_{\text {exc }}(r)$, or equivalently a higher level of confidence in the domain $G(r)$. The Chebychev inequalities ensure that $b_{2 n}(r)$ is an upper bound for $P_{\text {exc }}(r)$, regardless of the distribution of $\gamma$, while it is impossible to ascertain whether $P_{\text {Gauss }}(r)$ is an upper or lower bound for $P_{\text {exc }}(r)$.

\section{MAXIMUM-ENTROPY PRINCIPLE}

Beyond the Chebychev bounds, the most complete way of quantifying the randomness of $\gamma$ is to determine the pdf of $\gamma$. This can be achieved by resorting to a Maximum-Entropy (MaxEnt) method [14], which is a robust means to approximate the yet unknown pdf $f_{\gamma}$ starting from the statistical moments of $\gamma$. In this heuristic approach, every moment in $\mathcal{M}(M)$ is regarded as the result of the evaluation of a functional $\psi_{k}$ on $f_{\gamma}$, with

$$
\psi_{k}\left[f_{\gamma}\right]=\int_{\mathcal{G}} x^{k} f_{\gamma}(x) d x, \text { for } k=0, \ldots, M
$$


The set $\mathcal{M}(M)$ represents the totality of our knowledge about $f_{\gamma}$. This knowledge can be quantified by Shannon's entropy functional [13, p. 654],

$$
\mathcal{S}[f]=-\int_{\mathcal{G}} f(x) \ln [f(x)] d x, \quad \text { for } f \in \mathcal{L}^{1}\left(\mathcal{G}, \mathbb{R}_{+}\right),
$$

where $\mathcal{L}^{1}\left(\mathcal{G}, \mathbb{R}_{+}\right)$is the Lebesgue space of positive-valued measurable functions defined on $\mathcal{G}$. The MaxEnt method states that among all the possible pdfs that are compatible with the constraints gathered in $\mathcal{M}(M)$, the least biased pdf is the one that maximizes the entropy $\mathcal{S}$. Hence, the MaxEnt pdf is found by solving the optimization problem

$$
f_{\gamma, M}=\underset{\substack{\arg \max \\ f \in \mathcal{L}^{1}\left(\mathcal{G}, \mathbb{R}_{+}\right)}}{\mathcal{S}[f],}
$$

which is handled via Lagrange's multiplier method [27, p. 195] and yields the following density function [28]

$$
f_{\Lambda, M}(x)=\exp \left(-\sum_{k=0}^{M} \lambda_{k} x^{k}\right), \quad \text { for any } x \in \mathbb{R} .
$$

The particular form of $f_{\Lambda, M}$ in Eq. (15) leads to smooth pdfs, with e.g., $f_{\Lambda, 1}$ being an exponential pdf, and $f_{\Lambda, 2}$ a Gaussian pdf.

To obtain the Lagrange factors $\Lambda=\left(\lambda_{0}, \ldots, \lambda_{M}\right)$, Eq. (15) is inserted into the constraints expressed by Eq. (12), i.e.,

$$
\mu_{k}=\psi_{k}\left[f_{\Lambda, M}\right], \quad \text { for } k=0, \ldots, M,
$$

and the resulting system of nonlinear equations is solved for $\Lambda$ via a least-squares nonlinear regression algorithm [29, p. 607].

Equations (16) and (12) highlight the importance for the MaxEnt technique of the evaluation of integrals over $\mathcal{G}$, which is however unknown. An alternative would consist in evaluating the integrals over $\mathbb{R}$ rather than $\mathcal{G}$. However, the resulting improper integrals would be tedious to evaluate numerically, which in turn would hinder the solution of Eqs. (16) by nonlinear regression. Conversely, if these integrals are computed over a domain that is smaller than $\mathcal{G}$, the mismatch between the moments $\mathcal{M}(M)$ and the integrals $\psi_{k}\left[f_{\Lambda, M}\right]$ could produce false MaxEnt pdfs.

The alternative followed in this article employs the Chebychev confidence intervals $G(r)$, discussed in Section 3, as the domains of integration. Given a threshold $P_{\mathrm{thr}} \in[0,1]$, the radius $r_{\mathrm{thr}}$ of the confidence interval of level $P_{\mathrm{thr}}$ is the minimum value of $r$ such that $P_{\text {exc }}(r) \leq P_{\mathrm{thr}}$, i.e.,

$$
r_{\mathrm{thr}}=\min \left\{r>0, P_{\mathrm{exc}}(r) \leq P_{\mathrm{thr}}\right\} .
$$


From the inequality (9), upper bounds for $r_{\text {thr }}$ can be obtained as

$$
r_{2 n, \mathrm{thr}}=\left(\frac{\kappa_{2 n}}{P_{\mathrm{thr}}}\right)^{1 / 2 n}
$$

By limiting $P_{\text {thr }}$ to a sufficiently small value, the corresponding Chebychev confidence intervals $G\left(r_{2 n \text {,thr }}\right)$ will capture $\mathcal{G}$ without being unnecessarily large domains of integration. Thus, using the Chebychev bounds, the MaxEnt method can be applied in an adaptive manner with respect to the range of the observable. To the best of the authors' knowledge, this is the first stochastic approach which uses Chebychev's inequalities in conjunction with a Maximum-Entropy scheme.

The general steps followed in our stochastic rationale are summarized in Fig. 2.

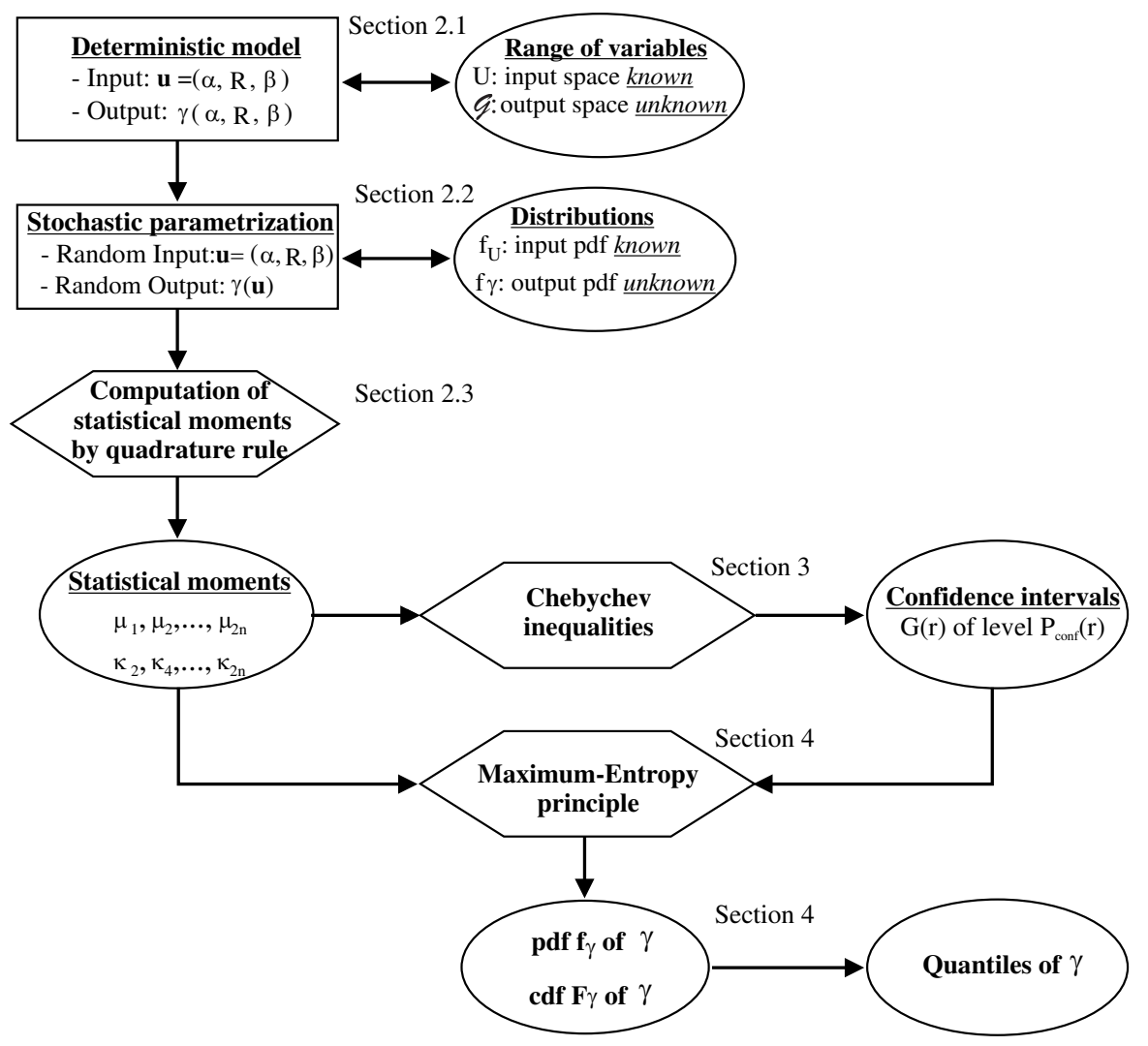

Figure 2. Summary of the stochastic approach using Chebychev's inequalities and the Maximum-Entropy principle. 


\section{VOLTAGE INDUCED AT THE PORT OF A THIN-WIRE SETUP}

\subsection{Configuration}

The methods presented in the Sections $2-4$ are now applied to study the interaction between a PEC thin-wire setup and an incident plane wave. With reference to Fig. 1 , the axis of the wire $\Omega_{\alpha}$ is parameterized by its Cartesian coordinates $\left[x_{\alpha}(y), y, z_{\alpha}(y)\right]$, with

$$
\begin{aligned}
& x_{\alpha}(y)=\alpha^{x} \sin \left(\pi \frac{y-y_{m}}{y_{M}-y_{m}}\right), \text { in meters, } \\
& z_{\alpha}(y)=0.05+\alpha^{z} \sin \left(\pi \frac{y-y_{m}}{y_{M}-y_{m}}\right), \text { in meters, }
\end{aligned}
$$

for any $y \in\left[y_{m} ; y_{M}\right]$, where $\alpha^{x} \in \mathcal{A}^{x}=[-0.02 ; 0.02] \mathrm{m}$ and $\alpha^{z} \in \mathcal{A}^{z}=\mathcal{A}^{x}$. This setup is connected to a resistance $R \in \mathcal{R}=[0,200] \Omega$. The geometrical model assumed in Eq. (19) can be encountered in periodic setups used for instance as filters [30].

The incident electric field $\mathbf{E}_{\beta}^{i}$ is a plane wave, which propagates along the direction $\left(\theta=45^{\circ}, \phi=45^{\circ}\right)$ in a spherical coordinate system, and which has an amplitude normalized to $1 \mathrm{~V} \cdot \mathrm{m}^{-1}$. The polarization $\psi$ of $\mathbf{E}_{\beta}^{i}$ varies in the interval $\mathcal{P}=\left[0^{\circ}, 90^{\circ}\right]$, with $\psi=0^{\circ}$ and $\psi=90^{\circ}$ corresponding to parallel- and perpendicular-polarized fields, respectively.

The observable $\gamma=\left|V_{e}\right|$ is computed via Eq. (1), where the current distribution $\mathbf{J}_{\alpha, R}$ is obtained by solving an EFIE. For efficiency purposes, a reduced-kernel EFIE [22] is employed together with quadratic-segment basis functions that are well suited to represent curved geometries [31]. In the present case, the surface of $\Omega_{\alpha}$ is meshed into 224 elements for use in the method of moments (MoM). The mesh of the straight wire $\Omega_{\alpha=0}$ comprises segments that are $5 \mathrm{~mm}$ long. Using a $2.4 \mathrm{GHz}$ computer with an Intel Core i3 processor, a single evaluation of $\left|V_{e}\right|$ requires $145 \mathrm{~ms}$, of which $115 \mathrm{~ms}$ are devoted to the construction and the solution of the MoM matrix.

\subsection{Motivation for the Numerical Examples}

To illustrate the significant effects that the uncertainties of the setup have on the observable, the spectrum of the voltage induced at the port of a straight wire $\Omega_{\boldsymbol{\alpha}=0}$ is plotted in Figs. 3(a) and 3(b) for a paralleland a perpendicular-polarized $\mathbf{E}_{\boldsymbol{\beta}}^{i}$, respectively. The frequency is swept from $f=100 \mathrm{MHz}$ (wavelength $\lambda \approx 3 \mathrm{~m}$ ) to $f=500 \mathrm{MHz}(\lambda \approx 0.6 \mathrm{~m}$ ). Lower or higher frequencies could be studied as well, provided that the 
deterministic model is accurate. Particularly at higher frequencies, one has to make sure that the mesh of the thin wire remains fine enough compared to the wavelength $\lambda$, e.g., with elementary segments smaller than $\lambda / 10 \approx 6 \mathrm{~cm}$. Besides these deterministic limitations, the stochastic method described in this article holds regardless of the frequency, i.e., from $\mathrm{kHz}$ frequencies encountered, e.g., in powerelectronics applications, to $\mathrm{GHz}$ or higher frequencies used in remotesensing and telecommunications applications.

The graphs in Figs. 3(a) and 3(b) clearly show the differences in the values of $\left|V_{e}\right|$ for $R \in\{0 \Omega, 50 \Omega, 200 \Omega\}$ as can be seen through the shift between the resonance frequencies of the short-circuit case $R=0 \Omega$ and the case $R=200 \Omega$. At a frequency such as $f=200 \mathrm{MHz}$, the variations of $R$ produce more than two orders of magnitude of changes in $\left|V_{e}\right|$. Compared to the short-circuit case $R=0 \Omega$, higher values of $R$ damp the peak value of $\left|V_{e}\right|$ observed at resonance frequencies. This damping effect is due to the power dissipation that occurs in the resistance $R$, unlike the short-circuit case where the traveling waves are reflected at the ends of the transmission line [21]. Moreover, the values of $\left|V_{e}\right|$ vary with the polarization of $\mathbf{E}_{\beta}^{i}$. Therefore, the uncertainty of the setup cannot be neglected.

In the remainder of this article, we focus our analysis on the following three frequencies,

- $f_{1}=280 \mathrm{MHz}$, which is a resonance frequency for $R=200 \Omega$;

- $f_{2}=545 \mathrm{MHz}$, which is a non-resonance frequency; and

- $f_{3}=900 \mathrm{MHz}$, which is a resonance frequency for $R=0 \Omega$.

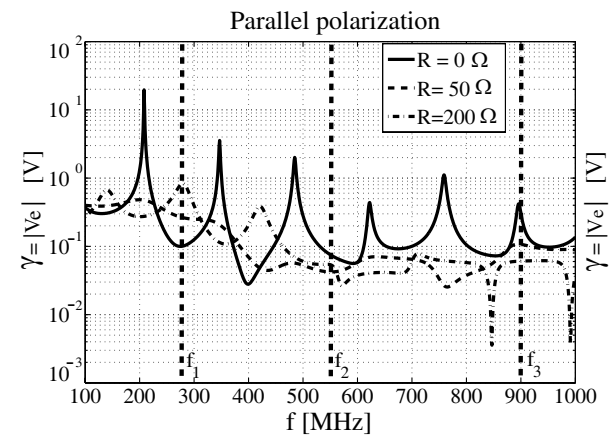

(a)

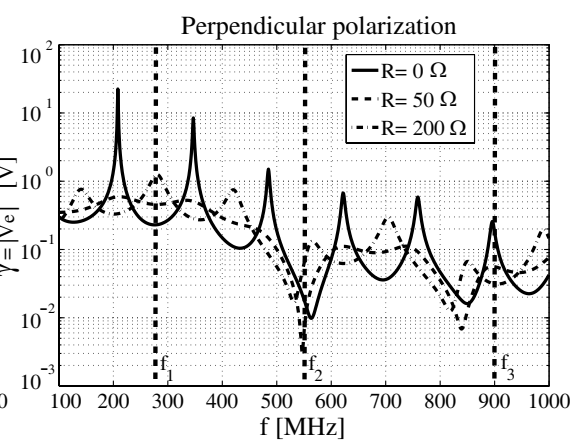

(b)

Figure 3. (a) Voltage $\left|V_{e}\right|$ induced by a parallel-polarized $\mathbf{E}_{\beta}^{i}$, i.e., $\psi=0^{\circ}$. (b) A perpendicular-polarized $\mathbf{E}_{\beta}^{i}$, i.e., $\psi=90^{\circ}$. Frequencies $f_{1}=280 \mathrm{MHz}, f_{2}=545 \mathrm{MHz}$ and $f_{3}=900 \mathrm{MHz}$, where the stochastic analysis will be conducted. 
The study of these frequencies will allow us to study the effect of the frequency on the uncertainty of the observable. In particular, a comparison of the resonances at $f_{1}$ and $f_{3}$ will show whether the variations of the setup and the incident field lead to similar distributions for the induced voltage.

\subsection{Randomization}

With the notations of Subsection 2.2, the vector of random input parameters is defined as $\mathbf{u}=\left(\alpha^{x}, \alpha^{z}, R, \psi\right)$ and it belongs to the domain $\mathcal{U}=\mathcal{A}^{x} \times \mathcal{A}^{z} \times \mathcal{R} \times \mathcal{P}$. The vector $\mathbf{u}$ is assumed to be random in $\mathcal{U}$ according to a uniform probability distribution $P_{\mathcal{U}}$. The components of $\mathbf{u}$ are assumed to be mutually statistically independent.

The general steps sketched in Fig. 2 are now followed to propagate the randomness of the input $\mathbf{u}$ through the electromagnetic model and to quantify the uncertainty of the observable $\left|V_{e}\right|$.

\subsection{Computation of Statistical Moments}

The first 14 statistical moments of $\left|V_{e}\right|$ are computed following the rationale presented in Subsection 2.3. To properly handle the various multi-dimensional integrals that are computed, a Monte-Carlo (MC) rule is used. As explained in Subsection 2.3, nested grids are used in the quadrature algorithm for numerical-efficiency purposes, which implies that the complexity is $N \equiv 2^{\ell}+1$. The accuracy of the quadrature rule is then evaluated for each of the 14 integrals that define the moments of $\left|V_{e}\right|$, by comparing the relative variation of the quadrature approximation as the complexity increases from $2^{\ell}+1$ to $2^{\ell+1}+1$. The maximum number of function evaluations is set at $N_{\max }=10^{5}$.

Figure 4 displays the complexity $N_{\left|V_{e}\right|}$ required to compute the statistical moments with a target maximum relative error $E_{\max }=10^{-2}$. The complexity is shown for the evaluation of the moments about the origin $\mu_{m}$ and for the evaluation of the normalized moments $\kappa_{m}$. The computation of higher-order moments implies a larger complexity as indicated by the increase of $N_{\left|V_{e}\right|}(m)$ with the order $m$ of the moment $\mu_{m}$. Overall, computing the moments about the origin is faster than the evaluation of the normalized moments, particularly for odd orders $m$. This difference in performance is due to the fact that the integrands defining odd normalized moments (see Eq. (10)) are centered about the origin and they take positive as well as negative values, which in turn slows down the convergence of the quadrature rule.

The mean $\mu_{1}$, the standard deviation $\sigma$ and the kurtosis $\kappa_{4}$ of $\left|V_{e}\right|$ are listed in Table 1 as diagnostic moments. The values of the kurtosis 

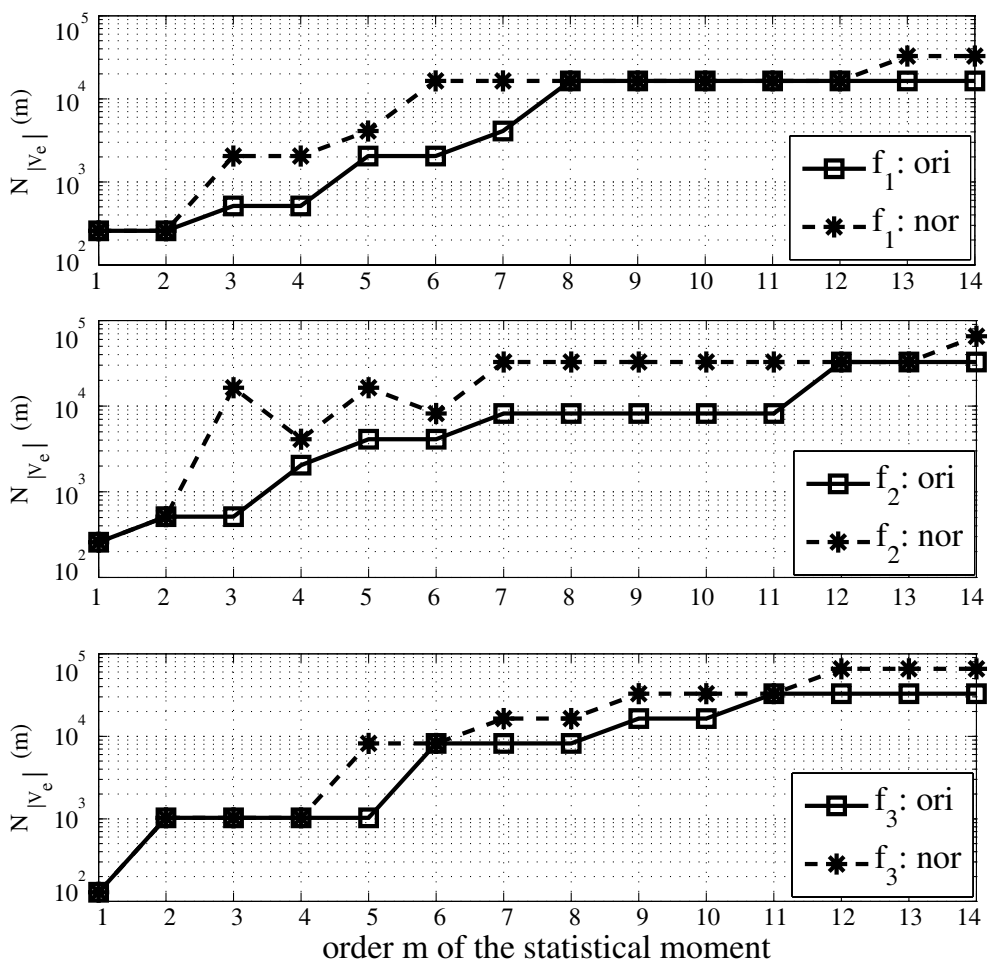

Figure 4. Complexity $N_{\left|V_{e}\right|}$ of the Monte-Carlo rule to compute the first 14 statistical moments of $\left|V_{e}\right|$ with a relative accuracy of $10^{-2}$. Moments about the origin (ori) and normalized moments (nor). Results for the frequencies $f_{1}=280 \mathrm{MHz}$ (top), $f_{2}=545 \mathrm{MHz}$ (middle) and $f_{3}=900 \mathrm{MHz}$ (bottom).

are given both for the case where $\kappa_{4}$ is evaluated directly by quadrature $\left(\kappa_{4}^{\text {nor }}\right)$ and for the case where it is deduced from the moments about the mean $\left(\kappa_{4}^{\text {ori }}\right)$ via Eq. $(10)$. Table 1 also contains the reference values of the statistical moments, which are obtained from a larger dataset that comprises $4 \times 10^{5}$ samples of the observable.

This table confirms the accuracy of the computation of the statistical moments and it provides some insight in the distribution of $\left|V_{e}\right|$ at $f_{1}, f_{2}$ and $f_{3}$. The frequency $f_{2}$ is characterized by a high concentration of the values of $\left|V_{e}\right|$ around their mean value as indicated by the small values of $\sigma\left(f_{2}\right)$ and $\kappa_{4}\left(f_{2}\right)$. The first resonance produces the largest value of $\sigma$, even though $\kappa_{4}\left(f_{1}\right)$ is slightly larger than 3 thereby implying a slightly wider spread than a Gaussian spread. Even though $\sigma\left(f_{3}\right)$ is three times smaller than $\sigma\left(f_{2}\right)$, the fact that $\kappa_{4}\left(f_{3}\right)$ is 
Table 1. Diagnostic statistical moments of $\left|V_{e}\right|$.

\begin{tabular}{c||c|c|c}
$n$ & $f_{1}$ & $f_{2}$ & $f_{3}$ \\
\hline$\mu_{1}^{\text {ref }}[V]$ & 0.335 & 0.031 & 0.108 \\
$\mu_{1}[V]$ & 0.335 & 0.031 & 0.108 \\
\hline$\sigma^{\text {ref }}[V]$ & 0.264 & 0.018 & 0.088 \\
$\sigma[V]$ & 0.264 & 0.018 & 0.088 \\
\hline$\kappa_{4}^{\text {rof }}$ & 3.88 & 2.49 & 9.38 \\
$\kappa_{4}^{\text {nor }}$ & 3.88 & 2.45 & 9.38 \\
$\kappa_{4}^{\text {ori }}$ & 3.90 & 2.53 & 9.38
\end{tabular}

conversely 2.5 times larger than $\kappa_{4}\left(f_{2}\right)$ indicates the presence of more outliers at $f_{3}$ than at $f_{2}$ and $f_{1}$, as explained in $[12,32]$.

\subsection{Higher-order Chebychev Bounds}

The higher-order Chebychev bounds are displayed in Figs. 5-7, together with the Gaussian estimate $P_{\text {Gauss }}$ discussed in Section 3. To serve as a reference, the actual exceedance probability $P_{\text {exc, ref }}(r)$ is estimated empirically using an ensemble of $N_{\text {det }}=4 \times 10^{5}$ voltage samples obtained from configurations that are drawn randomly via a random-number generator. The reference and Gaussian values of $P_{\text {exc }}$ are obtained from Eq. (6) where $F_{\gamma}$ is replaced by the empirical cdf $F_{\text {ref }}$ and the Gaussian cdf $F_{\text {Gauss }}$, respectively.

These graphs point out the conservative nature of $P_{\text {Gauss }}$ as opposed to $b_{2}$ which is very loose. The slopes of higher-order bounds become steeper as the order of the normalized moments increases. Higher-order Chebychev bounds become meaningful, i.e., below 1, later than lower-order bounds, as expected from Lyapunov's inequality (11). At $f_{1}$, for instance, if $r=2$, the only bounds that are lower than 1 and hence exploitable are $b_{2}, b_{4}$ and $b_{6}$.

The interpretation of the kurtosis given in the preceding section is confirmed by these plots, i.e., the larger the value of $\kappa_{4}$, the wider the spread of $\left|V_{e}\right|$, measured in terms of $\sigma$. Accordingly, the Gaussian bound slightly underestimates the actual spread at $f_{1}$ and overestimates the spread at $f_{2}$. At $f_{3}, P_{\text {Gauss }}$ clearly underestimates $P_{\text {exc }}$ once $r \geq 2$ and fails to account for the larger spread of $\left|V_{e}\right|$.

Moreover, $b_{2}$ is insensitive to the randomness of the observable, as expected from Eq. (8). Conversely, the higher-order bounds follow the randomness of the observable as can be seen for the low spread at $f_{2}$, and for the large spread at $f_{3}$. 


\subsection{Dimensioning Application}

The bounds in Figs. 5-7 are now employed for dimensioning purposes to estimate the range of $\left|V_{e}\right|$ via confidence intervals. More specifically, the objective is to estimate the $99.99 \%$ confidence interval, i.e., the domain with an exceedance probability of $0.01 \%$, which is defined as

$$
G\left(r_{0.01 \%}\right)=\left[0 ; V_{0.01 \%}\right] \text {, with } V_{0.01 \%}=\mu_{1}+r_{0.01 \%} \times \sigma,
$$

where $r_{0.01 \%}$ is determined from Eq. (18). The values of $r_{0.01 \%}$ and $V_{0.01 \%}$ are listed in Table 2 .

The Chebychev bounds lead to an over-dimensioning that is particularly broad when only two moments are used. The bounds improve significantly with higher-order moments. In contrast, the Gaussian bounds do not offer any guarantee as to whether they underor over-estimate the spread of the variable, as shown by the values obtained at $f_{1}$ and $f_{3}$.

The significance of Table 2 can be understood, e.g., in shielding applications where the objective is to protect any device connected at the port of the wire from large induced interferences. Assuming that $\left|V_{e}\right|$ follows a Gaussian distribution would lead to an insufficient protection against the induced perturbation at the resonance frequencies $f_{1}$ and $f_{3}$. On the contrary, once six or more moments are employed in Chebychev's inequality, accurate upper estimates of the range of $\left|V_{e}\right|$ are obtained.

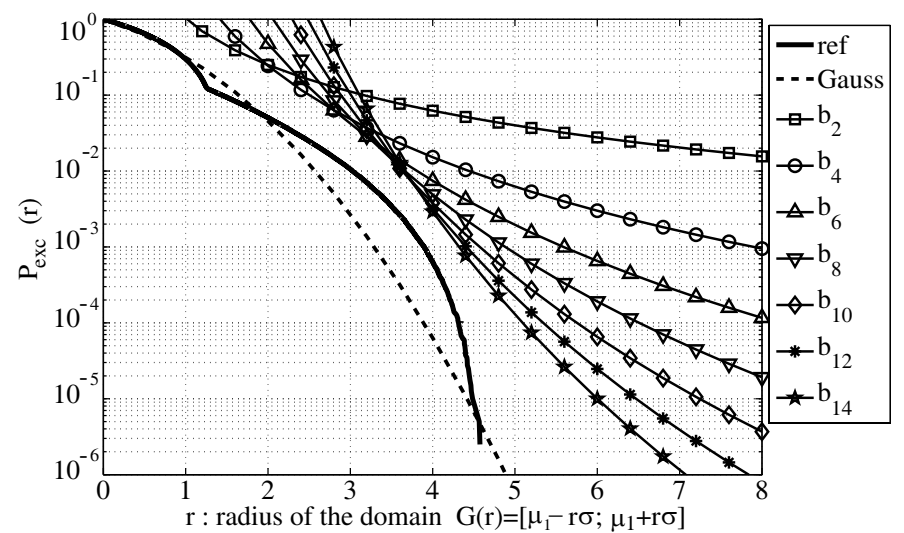

Figure 5. Frequency $f_{1}$ : Exceedance probability $P_{\mathrm{exc}}(r)=$ $P_{\gamma}\left[\left|\gamma-\mu_{1}\right|>r \sigma\right]$ : Reference value (ref), Gaussian estimate (Gauss), Chebychev bounds $\left(\left\{b_{2 m}\right\}_{m=1, \ldots, 7}\right)$. 


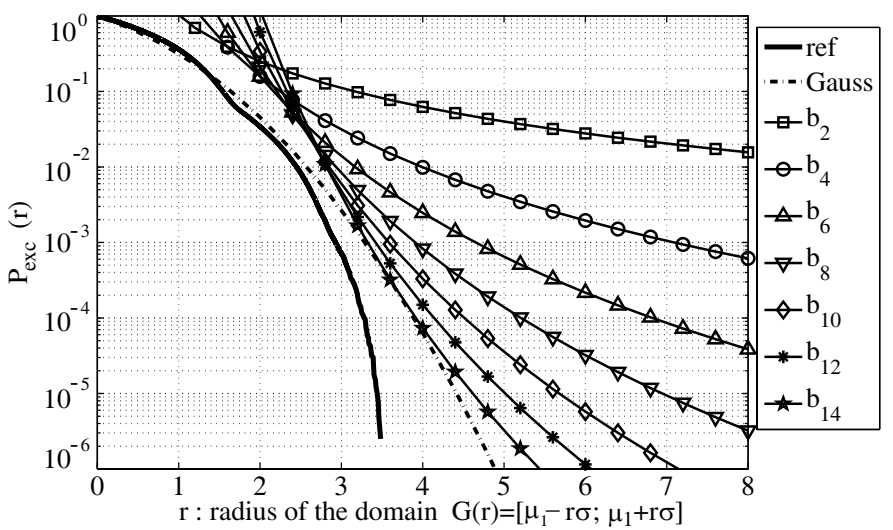

Figure 6. Frequency $f_{2}$ : Exceedance probability $P_{\text {exc }}(r)$ : Reference value (ref), Gaussian estimate (Gauss), Chebychev bounds $\left(\left\{b_{2 m}\right\}_{m=1, \ldots, 7}\right)$.

Table 2. Estimation of the range of $\left|V_{e}\right|$ via $99.99 \%$ confidence interval.

\begin{tabular}{c||c|c||c|c||c|c}
\multicolumn{1}{c||}{} & \multicolumn{2}{c||}{$f_{1}$} & \multicolumn{2}{c||}{$f_{2}$} & \multicolumn{2}{c}{$f_{3}$} \\
\cline { 2 - 7 } & $r_{0.01 \%}$ & $V_{0.01 \%}$ & $r_{0.01 \%}$ & $V_{0.01 \%}$ & $r_{0.01 \%}$ & $V_{0.01 \%}$ \\
\hline \hline Ref. & 4.3 & $1.475 \mathrm{~V}$ & 3.3 & $0.089 \mathrm{~V}$ & 5.0 & $0.548 \mathrm{~V}$ \\
\hline Gauss. & 3.9 & $1.365 \mathrm{~V}$ & 3.9 & $0.099 \mathrm{~V}$ & 3.9 & $0.451 \mathrm{~V}$ \\
\hline$b_{2}$ & 100 & $26.750 \mathrm{~V}$ & 100 & $1.792 \mathrm{~V}$ & 100 & $8.900 \mathrm{~V}$ \\
$b_{4}$ & 14.1 & $4.059 \mathrm{~V}$ & 12.6 & $0.253 \mathrm{~V}$ & 17.6 & $1.655 \mathrm{~V}$ \\
$b_{6}$ & 8.2 & $2.500 \mathrm{~V}$ & 6.8 & $0.150 \mathrm{~V}$ & 10.6 & $1.040 \mathrm{~V}$ \\
$b_{8}$ & 6.6 & $2.078 \mathrm{~V}$ & 5.2 & $0.122 \mathrm{~V}$ & 8.3 & $0.838 \mathrm{~V}$ \\
$b_{10}$ & 5.8 & $1.867 \mathrm{~V}$ & 4.5 & $0.110 \mathrm{~V}$ & 7.3 & $0.750 \mathrm{~V}$ \\
$b_{12}$ & 5.4 & $1.761 \mathrm{~V}$ & 4.2 & $0.105 \mathrm{~V}$ & 6.7 & $0.697 \mathrm{~V}$ \\
$b_{14}$ & 5.1 & $1.682 \mathrm{~V}$ & 3.9 & $0.099 \mathrm{~V}$ & 6.3 & $0.662 \mathrm{~V}$
\end{tabular}

\subsection{Maximum-entropy Results}

The MaxEnt method described in Section 4 is now applied. The nonlinear regression to compute the Lagrange multipliers is performed using a Levenberg-Marquardt algorithm [27, p. 100] (function "nlinfit" in MATLAB $(\mathbb{B})$. First, focusing on the frequency $f_{1}$, the effect on the MaxEnt pdfs of the choices made about the range of $\left|V_{e}\right|$ are illustrated. Next, the refinement of the MaxEnt results by higher-order moments is demonstrated at $f_{2}$ and $f_{3}$. Lastly, the MaxEnt pdfs are used to determine the cdf of $\left|V_{e}\right|$, from which quantiles are deduced. 


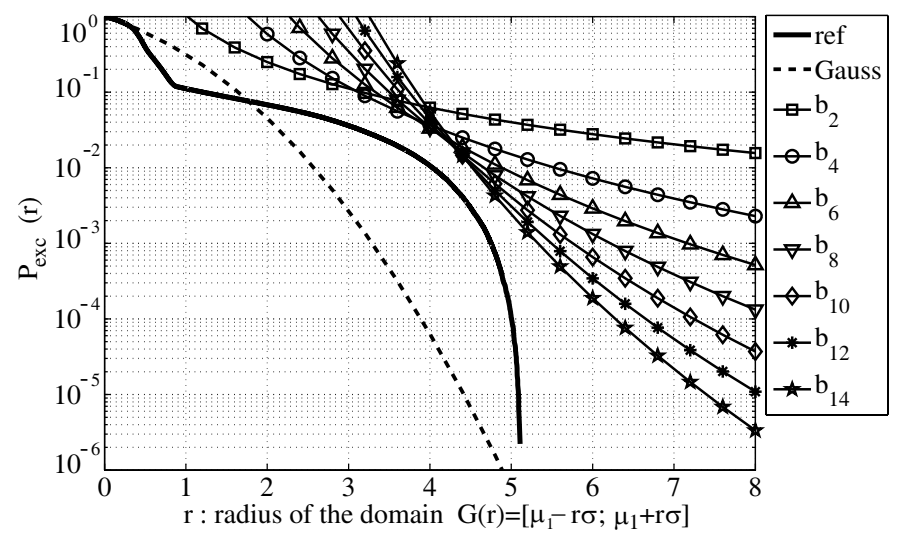

Figure 7. Frequency $f_{3}$ : Exceedance probability $P_{\text {exc }}(r)$ : Reference value (ref), Gaussian estimate (Gauss), Chebychev bounds $\left(\left\{b_{2 m}\right\}_{m=1, \ldots, 7}\right)$.

\subsubsection{Importance of the Range of $\left|V_{e}\right|$}

The first six moments of $\left|V_{e}\right|$, at the frequency $f_{1}=245 \mathrm{MHz}$, are used to compute the MaxEnt pdf $f_{\Lambda, 6}$. In the MaxEnt algorithm, the range of $\left|V_{e}\right|$ used in the integrals of Eq. (16) is chosen as $G(r)=\left[0 ; \mu_{1}+r \sigma\right]$, with $\mu_{1}=0.335 \mathrm{~V}, \sigma=0.264 \mathrm{~V}$ and $r \in\{3,4,5,8\}$. According to Fig. $5, G(3)$ and $G(4)$ are subsets of the actual range $\mathcal{G}=[0 ; 1.519] \mathrm{V}$, whereas $G(5)$ and $G(8)$ are wider than $\mathcal{G}$. Moreover, based on Table 2, the $99.99 \%$ confidence intervals obtained assuming a Gaussian distribution for $\left|V_{e}\right|$ would be $G(3.9) \approx G(4)$, while the Chebychev bound $b_{6}$ would lead approximately to the interval $G(8.2) \approx G(8)$. Fig. 8 depicts $f_{\Lambda, 6}$ for the different supports $G(r)$, with $r \in\{3,4,5,8\}$. The severe consequences of an underestimation of $\mathcal{G}$ can be seen through the pdf obtained using $G(3)$. The pdf obtained with $G(4)$ is accurate over $G(4)$ but diverges once $\left|V_{e}\right|$ exceeds the upper limit of this interval. For $G(5)$ and $G(8)$, the resulting pdfs are accurate when compared to $f_{\text {ref }}$ over the entire domain $\mathcal{G}$. Hence, the overdimensioning through Chebychev's bounds is an advantage for the computation of the MaxEnt pdf.

\subsubsection{Increasing the Number of Moments}

To illustrate the refinement of the MaxEnt pdfs via higher-order moments, the pdfs $f_{\Lambda, 4}$ and $f_{\Lambda, 8}$ are computed at $f_{2}=545 \mathrm{MHz}$. The range of $\left|V_{e}\right|$ is chosen according to Table 2 by using first the Gaussian estimate which leads to $G(3.9)=[0 ; 0.099] \mathrm{V}$, and the Chebychev 
bound $b_{8}$, which leads to $G(5.2)=[0 ; 0.122] \mathrm{V}$. The results are plotted in Fig. 9.

Since both $G(3.9)$ and $G(5.2)$ contain the range of $\left|V_{e}\right|$, which is $\mathcal{G}=[0 ; 0.091] \mathrm{V}$, both choices lead to accurate MaxEnt pdfs. With reference to $f_{\text {ref }}, f_{\Lambda, 4}$ and $f_{\Lambda, 8}$ succeed in capturing the support of the pdf of $\left|V_{e}\right|$. Compared to $f_{\Lambda, 4}$, the use of higher-order statistical information in $f_{\Lambda, 8}$ enables it to precisely reproduce the slope of $f_{\text {ref }}$, particularly for $\left|V_{e}\right| \leq 0.05 \mathrm{~V}$.

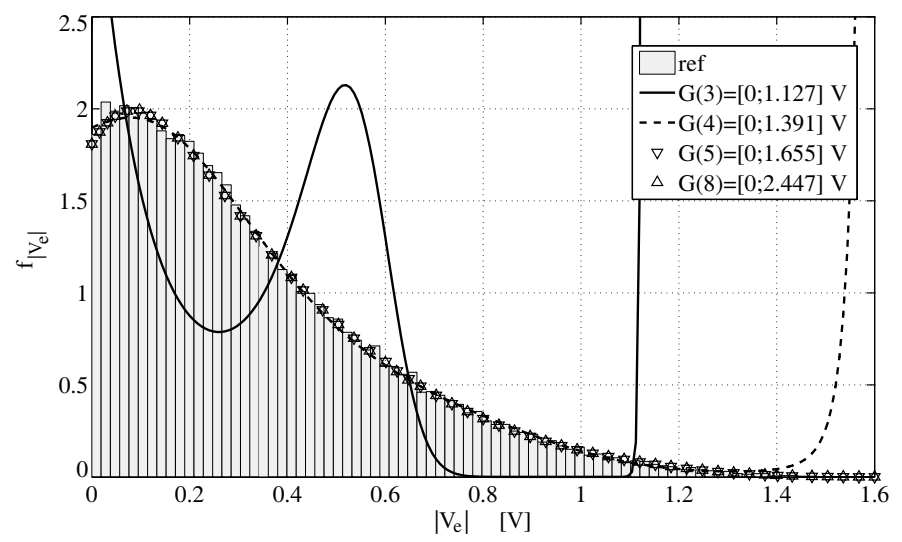

Figure 8. MaxEnt pdf of $\left|V_{e}\right|$ at $f_{1}=280 \mathrm{MHz}$, using $M=\{4,8\}$ statistical moments, versus the histogram $f_{\text {ref }}$ of $4 \times 10^{5}$ deterministic samples of $\left|V_{e}\right|$.

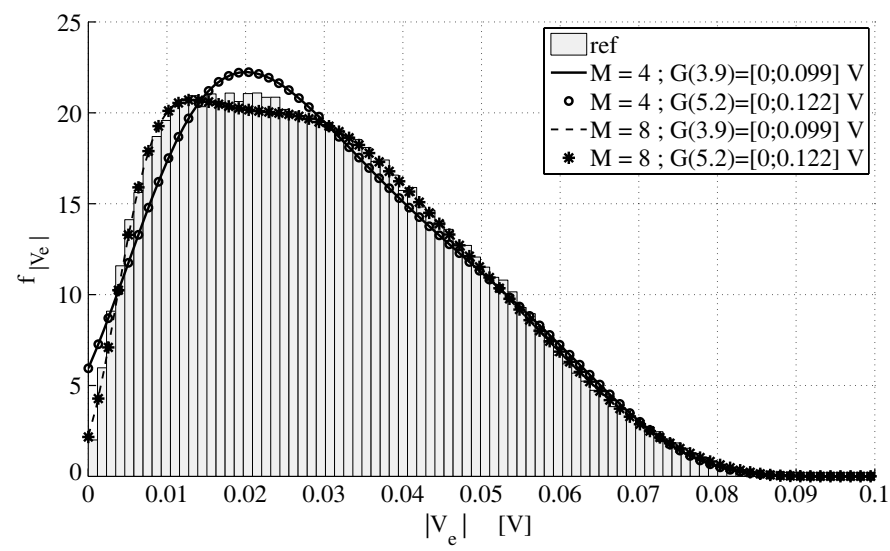

Figure 9. MaxEnt pdf of $\left|V_{e}\right|$ at $f_{2}=545 \mathrm{MHz}$, using $M=\{4,8\}$ statistical moments, versus the histogram $f_{\text {ref }}$ of $4 \times 10^{5}$ deterministic samples of $\left|V_{e}\right|$. 


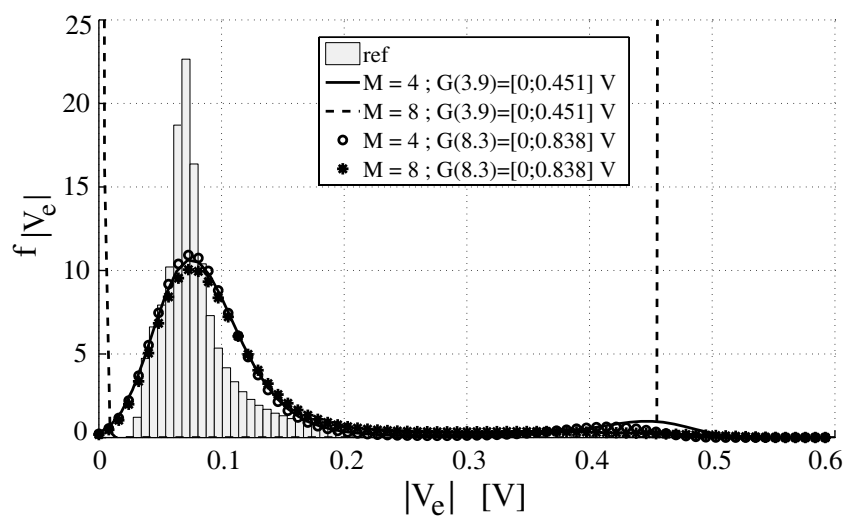

(a)

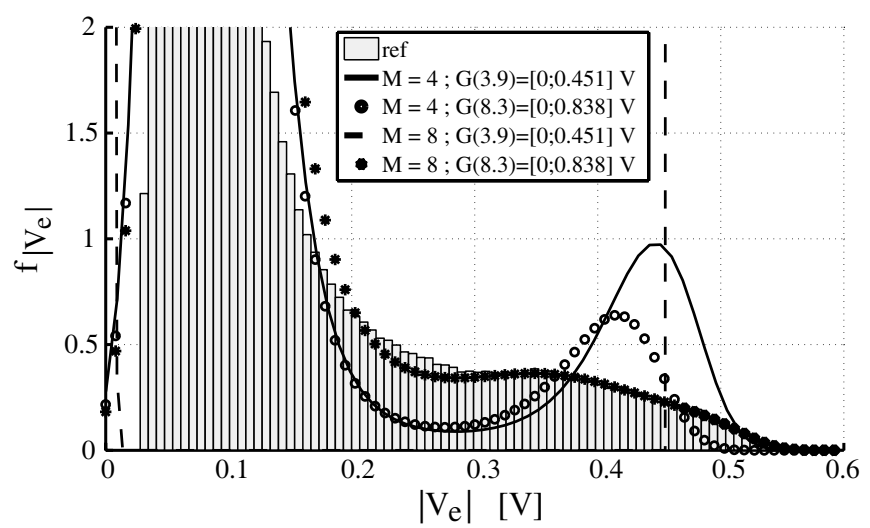

(b) Zoom in

Figure 10. MaxEnt pdf of $\left|V_{e}\right|$ at $f_{3}=900 \mathrm{MHz}$, using $M=\{4,8\}$ statistical moments, versus the histogram $f_{\text {ref }}$ of $4 \times 10^{5}$ deterministic samples of $\left|V_{e}\right|$.

The same procedure is applied at the frequency $f_{3}=900 \mathrm{MHz}$, where the range of the observable is now $\mathcal{G}=[0.029 ; 0.554] \mathrm{V}$. A Gaussian $99.99 \%$ confidence interval under-estimates $\mathcal{G}$ via $G(3.9)=$ $[0 ; 0.451] \mathrm{V}$, whereas $b_{8}$ over-estimates $\mathcal{G}$ via $G(8.3)=[0 ; 0.838] \mathrm{V}$. Figs. 10(a) and 10(b) show that the choices of $G(3.9)$ or $G(8.3)$ lead to slight changes in the resulting $f_{\Lambda, 4}$, which occur particularly around the tail of the pdf. For $f_{\Lambda, 8}$ however, the Gaussian under-estimate $G(3.9)$ prevents the MaxEnt algorithm from converging to a suitable pdf. On the contrary, with $G(8.3)$ the pdf $f_{\Lambda, 8}$ is accurate and approximates the tail of $f_{\text {ref }}$ more accurately than $f_{\Lambda, 4}$, as demonstrated by Fig. 10 (b). 
Table 3. Quantile $q_{99}$ of $\left|V_{e}\right|$ and relative error with respect to $q_{\text {ref }}$.

\begin{tabular}{c||c|c||c|c||c|c}
\multicolumn{1}{c||}{} & \multicolumn{3}{c||}{$f_{1}$} & \multicolumn{2}{c||}{$f_{2}$} & \multicolumn{2}{c}{$f_{3}$} \\
\cline { 2 - 7 } & $q_{99}[\mathrm{~V}]$ & $\varepsilon_{\text {rel }}$ & $q_{99}[\mathrm{~V}]$ & $\varepsilon_{\text {rel }}$ & $q_{99}[\mathrm{~V}]$ & $\varepsilon_{\text {rel }}$ \\
\hline \hline Ref. & 1.133 & - & 0.074 & - & 0.462 & - \\
\hline Gauss. & 0.949 & -0.162 & 0.073 & -0.014 & 0.313 & -0.323 \\
\hline$M=2$ & 1.136 & 0.003 & 0.076 & 0.027 & 0.380 & -0.178 \\
$M=3$ & 1.131 & -0.002 & 0.076 & 0.027 & 0.377 & -0.184 \\
$M=4$ & 1.131 & -0.002 & 0.073 & -0.014 & 0.445 & -0.037 \\
$M=5$ & 1.131 & -0.002 & 0.073 & -0.014 & 0.454 & -0.017 \\
$M=6$ & 1.137 & 0.004 & 0.074 & 0 & 0.459 & -0.007 \\
$M=7$ & 1.137 & 0.004 & 0.074 & 0 & 0.459 & -0.007 \\
$M=8$ & 1.137 & 0.004 & 0.074 & 0 & 0.464 & 0.004
\end{tabular}

The difficulty for $f_{\Lambda, 8}$ to converge when the support used in the MaxEnt algorithm is $G(3.9)$ is caused by the difficulty for the algorithm to find a suitable set of Lagrange coefficients that would produce a pdf with higher-order moments matching the computed higher-order moments. This example is yet another illustration of the advantage of resorting to the Chebychev bounds to estimate the range of the observable.

\subsubsection{Quantiles of $\left|V_{e}\right|$}

Once the pdf $f_{\left|V_{e}\right|}$ of $\left|V_{e}\right|$ has been accurately approximated by MaxEnt, the cumulative distribution function (cdf) $F_{\left|V_{e}\right|}$ can be obtained as follows

$$
F_{\left|V_{e}\right|}(v)=\int_{-\infty}^{v} f_{\left|V_{e}\right|}\left(v^{\prime}\right) d v^{\prime}, \quad \text { for any } v \in \mathbb{R} .
$$

This cdf is then used to evaluate the $99 \%$ quantile of $\left|V_{e}\right|$, i.e., the value $q_{99}$ of $\left|V_{e}\right|$, for which $F_{\left|V_{e}\right|}\left(q_{99}\right)=0.99$. The values of $q_{99}$ obtained from the MaxEnt pdf of $\left|V_{e}\right|$ are listed in Table 3 together with the quantiles $q_{\text {ref }}$ and $q_{\text {Gauss }}$ obtained from the cdf $F_{\text {ref }}$ and a Gaussian cdf $F_{\text {Gauss }}$, respectively.

The relative error between the estimated $q_{99}$ and the reference $q_{99}$ is also provided in Table 3. This metric shows the magnitude of the error made by assuming a Gaussian distribution over $\mathbb{R}$ for $\left|V_{e}\right|$, as evidenced by the large under-estimations of $q_{99}$ at $f_{1}$ and $f_{3}$. Although the MaxEnt cdf $F_{\Lambda, 2}$ is Gaussian, its definition also includes 
information about the range of $\left|V_{e}\right|$ via the MaxEnt algorithm. This additional item of information explains the better accuracy of $F_{\Lambda, 2}$, compared to $F_{\text {Gauss }}$, for the evaluation of $q_{99}$. The numbers in Table 3 also highlight the added value of higher-order moments in the MaxEnt algorithm, even at $f_{3}$, which involves a relatively rough pdf.

Overall, the MaxEnt results yield finer statistical information than the bounds given by Chebychev's inequality. In a design process, if the objective is to accurately estimate the dynamic range of $\left|V_{e}\right|$, the value of $q_{99}$ should be privileged. On the other hand, in a shielding application where the objective is to protect oneself form large induced voltages, the confidence intervals obtained via Chebychev's inequalities can be chosen as conservative upper bounds for the range of the induced voltage.

In a decision-making process where the uncertainty budget needs to be assessed, the results in Subsections 5.4-5.7 show that by assuming a Gaussian distribution for $\left|V_{e}\right|$, one can still obtain accurate results at the non-resonance frequency $f_{2}$. However, the limitations of such an assumption are clearly visible once resonances appear, i.e., at $f_{1}$ and $f_{3}$. The occurrence and intensity of resonances requires specific conditions both in terms of the electrical length of the thin wire and in the value of the impedance $R$ [19,p. 254-257], [11]. Hence, at $f_{1}$ and $f_{3}$, due to the uncertainty of the geometrical and physical properties of the wire and the polarization variations of the incident field, only a few configurations will produce resonances, i.e., large values of $\left|V_{e}\right|$. This explains the more pronounced right-skewness and the "heavier" tail of the pdf of $\left|V_{e}\right|$ at $f_{1}$ and $f_{3}$.

\section{CONCLUSION}

We have presented and illustrated a stochastic method to quantify uncertainties in computational electromagnetic engineering. This method, which is based on the computation and the use of higherorder moments of the observable, refines the traditional second-order Chebychev inequality and produces accurate upper bounds that are globally valid.

The study of the induced voltage of a thin-wire setup affected by geometrical and physical uncertainties has been conducted. The computation of higher-order moments comes at the expense of a larger complexity, even though the Monte-Carlo algorithm copes well with the increasing roughness of the integrals that define higher-order moments.

The thin-wire test case provides various examples of uncertainties owing to its resonance frequencies, which are highly sensitive to the actual configuration and produce significant modifications of the 
observable. In all these cases, the higher-order Chebychev bounds have proved to adapt well to the nature of the distribution of the observable, unlike a fitted Gaussian distribution. The availability of these bounds has a practical value for decision making processes such as exceedance probabilities or confidence intervals. Moreover, the conservative nature of the Chebychev bounds allows for the use, in a range-adaptive manner, of statistical post-processing tools such as the Maximum-entropy principle, as illustrated in this paper.

More generally, the results obtained in this article encourage the use of a two-stage approach wherein the higher-order moments are first used to compute Chebychev bounds and obtain confidence intervals. Next, these confidence intervals should be used in a MaxEnt scheme to accurately approximate the pdf and the cdf of the observable, thereby achieving a complete quantification of the stochastic uncertainties of the problem. The usefulness of these tools has been illustrated in an EMC context.

\section{ACKNOWLEDGMENT}

This work was funded by the Dutch Ministry of Economic Affairs, in the Innovation Research Program (IOP) number EMVT 04302.

\section{REFERENCES}

1. Balanis, C., "Antenna theory: A review," Proc. of the IEEE, Vol. 80, No. 1, 7-23, 1992.

2. Bruns, H. D., C. Schuster, and H. Singer, "Numerical electromagnetic field analysis for EMC problems," IEEE Trans. EMC., Vol. 49, No. 2, 253-262, 2007.

3. Bai, L., Z.-S. Wu, H.-Y. Li, and T. Li, "Scalar radiative transfer in discrete media with random oriented prolate spheroids particles," Progress In Electromagnetics Research B, Vol. 33, 21-44, 2011.

4. Moglie, F., V. M. Primiani, and A. P. Pastore, "Modeling of the human exposure inside a random plane wave field," Progress In Electromagnetics Research B, Vol. 29, 251-267, 2011.

5. Lang, R., M. Bahie-El Din, and R. Pickholtz, "Stochastic effects in adaptive null-steering antenna array performance," IEEE Journal on Selected Areas in Communications, Vol. 3, No. 5, 767-778, 1985.

6. Bellan, D. and S. Pignari, "Complex random excitation of electrically-short transmission lines," Proc. IEEE International Symposium on EMC, Vol. 3, 663-668, 2006. 
7. Hill, D., "Plane wave integral representation for fields in reverberation chambers," IEEE Trans. EMC., Vol. 40, No. 3, 209217, 1998.

8. Primiani, V. M. and F. Moglie, "Numerical simulation of LOS and NLOS conditions for an antenna inside a reverberation chamber," Journal of Electromagnetic Waves and Applications, Vol. 24, Nos. 17-18, 2319-2331, 2010.

9. Li, Z., L. L. Liu, and C. Q. Gu, "Generalized equivalent cable bundle method for modeling EMC issues of complex cable bundle terminated in arbitrary loads," Progress In Electromagnetics Research, Vol. 123, 13-30, 2012.

10. Xie, H., J. Wang, S. Li, H. Qiao, and Y. Li, "Analysis and efficient estimation of random wire bundles excited by plane-wave fields," Progress In Electromagnetics Research B, Vol. 35, 167-185, 2011.

11. Sy, O. O., M. C. van Beurden, B. L. Michielsen, J. A. H. M. Vaessen, and A. G. Tijhiuis, "Second-order statistics of complex observables in fully stochastic electromagnetic interactions: Applications to EMC," Radio Science, Vol. 45, RS4004.1-RS4004.16, 2010.

12. Sy, O. O., M. C. Van Beurden, B. L. Michielsen, J. A. H. M. Vaessen, and A. G. Tijhuis, "A statistical characterization of resonant electromagnetic interactions with thin wires: Variance and kurtosis analysis," Scientific Computing in Electrical Engineering (SCEE), 117-124, Springer-Verlag, Berlin, Heidelberg, 2010.

13. Papoulis, A. and S. U. Pillai, Probability, Random Variables and Stochastic Processes, Series in Electrical and Computer Engineering, McGraw-Hill, 2002.

14. Jaynes, E. T., "Information theory and statistical mechanics," Phys. Rev., Vol. 106, No. 4, 620-630, 1957.

15. Mrozynski, G., V. Schulz, and H. Garbe, "A benchmark catalog for numerical field calculations with respect to EMC problems," Proc. IEEE International Symposium on EMC, Vol. 1, 497-502, 1999.

16. Parmantier, J.-P., "Numerical coupling models for complex systems and results," IEEE Trans. EMC., Vol. 46, No. 3, 359$367,2004$.

17. Rumsey, V. H., "Reaction concept in electromagnetic theory," Phys. Rev., Vol. 94, No. 6, 1483-1491, 1954.

18. Michielsen, B. L. and C. Fiachetti, "Covariance operators, green functions, and canonical stochastic electromagnetic fields," Radio 
Science, Vol. 40, No. 5, RS5001.1-RS5001.12, 2005.

19. King, R., Transmission-Line Theory, Dover Publications, 1965.

20. Larrabee, D. A., "Reduced coupling of electromagnetic waves to transmission lines due to radiation effects," PIERS Proceedings, Pisa, Italy, March 28-31, 2004.

21. Larrabee, D. A., "Electromagnetic effects on transmission lines," International Conf. Software in Telecom. and Computer Networks (SoftCOM), 37-42, 2006.

22. Mei, K. K., "On the integral equation of thin wire antennas," IEEE Trans. Ant. Prop., Vol. 13, No. 3, 374-378, 1965.

23. Harrington, R., Field Computation by Moment Methods, Macmillan, New York, 1968.

24. Bharucha-Reid, A., Random Integral Equations, Vol. 96, R. Bellman, Ed., Mathematics in Science and Engineering, 1972.

25. Krommer, A. R. and C. W. Ueberhuber, Computational Integration, SIAM, Philadelphia, 1998.

26. Gerstner, T. and M. Griebel, "Numerical integration using sparse grids," Numerical Algorithms, Vol. 18, No 3, 209-232, 1998.

27. Fletcher, R., Practical Methods of Optimization, Wiley, 1987.

28. Einbu, J., "On the existence of a class of maximum-entropy probability density function," IEEE Trans. Info. Theory, Vol. 23, $772-775,1977$.

29. Graybill, F. and H. Iyer, Regression Analysis, Concepts and Applications, Duxbury Press, 1994.

30. Nitsch, J. B. and S. V. Tkachenko, "Propagation of highfrequency current waves along periodical thin-wire structures," Third International Conference on Ultrawideband and Ultrashort Impulse Signals, 279-281, 2006.

31. Champagne II, N. J., J. T. Williams, and D. R. Wilton, "The use of curved segments for numerically modeling thin wire antennas and scatterers," IEEE Trans. Ant. Prop., Vol. 40, No. 6, 682-689, 1992.

32. Sy, O. O., M. van Beurden, B. Michielsen, and A. Tijhuis, "Variance and kurtosis-based characterization of resonances in stochastic transmission lines: Local versus global random geometries," Turk. J. Elec. Eng. \& Comp. Sci., Vol. 17, No. 3, 2009 . 